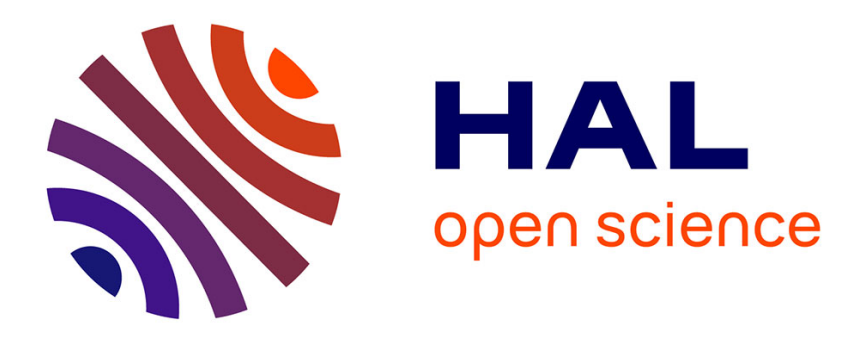

\title{
Formability prediction of thin metal sheets using various localization criteria
}

\author{
Guillaume Altmeyer, Farid Abed-Meraim, Tudor Balan
}

\section{To cite this version:}

Guillaume Altmeyer, Farid Abed-Meraim, Tudor Balan. Formability prediction of thin metal sheets using various localization criteria. International Journal of Material Forming, 2009, 2 (1), pp.423-426. 10.1007/s12289-009-0479-8 . hal-00753380

\section{HAL Id: hal-00753380 \\ https://hal.science/hal-00753380}

Submitted on 19 Nov 2012

HAL is a multi-disciplinary open access archive for the deposit and dissemination of scientific research documents, whether they are published or not. The documents may come from teaching and research institutions in France or abroad, or from public or private research centers.
L'archive ouverte pluridisciplinaire HAL, est destinée au dépôt et à la diffusion de documents scientifiques de niveau recherche, publiés ou non, émanant des établissements d'enseignement et de recherche français ou étrangers, des laboratoires publics ou privés. 


\title{
FORMABILITY PREDICTION OF THIN METAL SHEETS USING VARIOUS LOCALIZATION CRITERIA
}

\author{
G. Altmeyer ${ }^{*}$, F. Abed-Meraim, T. Balan \\ LPMM, Arts et Métiers ParisTech, Center of Metz, 4 rue A. Fresnel, 57078 Metz, France
}

\begin{abstract}
The aim of this paper is to give an overview of the theoretical basis of the most significant and commonly used localization criteria reformulated into a unified framework, and to apply these criteria to different materials in order to determine their formability domains.

After giving a general material description based on a phenomenological approach, theoretical foundations of localization criteria are presented together with their advantages and drawbacks. These criteria rely on diverse theories: maximum load principle, bifurcation analysis, Marciniak-Kuczynski analysis, and stability analysis by a linear perturbation method. They are then applied to a brass and a Dual Phase steel and the predicted Forming Limit Diagrams (FLD) are discussed.
\end{abstract}

KEYWORDS: Formability, Strain localization, Forming limit diagram.

\section{INTRODUCTION}

During sheet metal forming processes, plastic instability phenomena such as diffuse necking or strain localization may occur, leading to defective products. The development as well as the appropriate use of dimensioning methods to detect and avoid such phenomena are then of major interest for the sheet forming industry. The concept of Forming Limit Diagrams (FLDs) has been one of the earliest methods coined, and is still widely used nowadays to represent the formability of thin metal sheets. The FLDs can be obtained by various ways, including experimental methods, empirical localization indicators and theoretically based localization criteria. However, the comparison of FLDs obtained with these different methods is scarce, and it is still difficult to point out their respective ability to accurately determine the formability of a metal sheet.

The aim of this paper is to give an overview of the theoretical basis of the localization criteria based on the maximum load principle (Hill [1], Hora et al. [2]), on the bifurcation analysis (Drucker [3], Rice [4]), on the Marciniak-Kuczynski analysis [5] and on the stability analysis by a linear perturbation method (Dudzinski and Molinari [6]). The reformulation of these criteria into a general and unified framework allows the comparison of their theoretical formulation as well as the comparison of their numerical predictions in terms of both Forming Limit Diagrams.

\section{MATERIAL MODELLING}

\subsection{CONSTITUTIVE EQUATIONS}

The modelling adopted here is based on a phenomenological approach, used to describe the behaviour of elasto-plastic materials such as metal alloys. Since the material modelling was shown to have an influence on the Forming Limit Diagrams, initial and induced anisotropy as well as damage are introduced to accurately describe the material behaviour.

A hypo-elastic law is introduced:

$$
\dot{\boldsymbol{\sigma}}=\mathbf{C}: \mathbf{D}^{e}=\mathbf{C}:\left(\mathbf{D}-\mathbf{D}^{p}\right)
$$

where $\dot{\boldsymbol{\sigma}}$ is the Cauchy stress rate tensor and $\mathbf{D}, \mathbf{D}^{e}$, $\mathbf{D}^{p}$ are the total strain rate tensor and its elastic and plastic parts, respectively. $\mathbf{C}$ is the fourth-order elastic modulus tensor.

A yield function is given by (2):

$$
f\left(\boldsymbol{\sigma}, \mathbf{y}_{1}, \mathbf{y}_{2}, \ldots, \mathbf{y}_{n}\right) \leq 0, \dot{\lambda} \geq 0, \dot{\lambda} f=0
$$

where $\dot{\lambda}$ is the plastic multiplier. Variables $\mathbf{y}_{1}, \ldots, \mathbf{y}_{i}, \ldots, \mathbf{y}_{n}$ represent the set of internal variables, such as isotropic and kinematic hardening variables. The evolution of these internal variables is described by nonlinear differential laws, written under the following compact form:

$$
\dot{\mathbf{y}}_{i}=\dot{\lambda} \mathbf{H}_{\mathbf{y}_{i}}
$$

\footnotetext{
* Corresponding author: LPMM - Arts et Métiers ParisTech, Center of Metz, 4 rue A. Fresnel, 57078 Metz, France phone: +33-387-375-430; fax: +33-387-374-284; email address: guillaume.altmeyer@ metz.ensam.fr
} 
where $\mathbf{H}_{\mathbf{y}_{i}}$ is a modulus associated with the considered internal variable. The plastic flow rule is considered here for associative plasticity:

$$
\mathbf{D}^{p}=\dot{\lambda} \frac{\partial f}{\partial \boldsymbol{\sigma}}=\dot{\lambda} \mathbf{V}
$$

where $\mathbf{V}$ is the flow direction, normal to the yield surface defined by potential $f$. The combination of these equations allows the derivation of the tangent modulus $\mathbf{L}$ for an undamaged material:

$$
\dot{\boldsymbol{\sigma}}=\left\{\mathbf{C}-\frac{(\mathbf{C}: \mathbf{V}) \otimes(\mathbf{V}: \mathbf{C})}{\mathbf{V}: \mathbf{C}: \mathbf{V}-\sum_{i} \frac{\partial f}{\partial \mathbf{y}_{i}}: \mathbf{H}_{\mathbf{y}_{i}}}\right): \mathbf{D}=\mathbf{L}: \mathbf{D}
$$

For some steel alloys, damage may play a significant role. In this work, damage is introduced following the isotropic Continuum Damage Mechanics approach:

$$
\dot{\boldsymbol{\sigma}}=(1-d) \mathbf{C}:\left(\mathbf{D}-\mathbf{D}^{p}\right)-\frac{\dot{d}}{1-d} \boldsymbol{\sigma}
$$

with $d$ the damage variable, ranging from 0 for safe materials to 1 for fully damaged materials.

\subsection{MATERIAL PARAMETERS}

Classical quadratic Hill'48 yield surface is used to describe the initial anisotropy. For isotropic hardening, Swift power law is used for a brass, while Voce saturating law is used for a dual phase steel:

$$
\begin{aligned}
& \dot{R}_{\text {Swift }}=n k\left(\varepsilon_{0}+\bar{\varepsilon}\right)^{n-1} \dot{\lambda}=H_{R} \dot{\lambda} \\
& \dot{R}_{\text {Voce }}=C_{R}\left(R_{\text {sat }}-R\right) \dot{\lambda}=H_{R} \dot{\lambda}
\end{aligned}
$$

where $\bar{\varepsilon}$ is the cumulative plastic strain and $n, k, \varepsilon_{0}$, $C_{R}$ and $R_{\text {sat }}$ are material parameters. The evolution of the damage variable is governed by the law:

$$
\dot{d}= \begin{cases}\frac{1}{(1-d)^{\beta_{d}}}\left(\frac{Y_{e}-Y_{e i}}{S_{d}}\right)^{s_{d}} \dot{\lambda} & \text { if } Y_{e} \geq Y_{e i} \\ 0 & \text { otherwise }\end{cases}
$$

with $Y_{e}$ is the strain energy density release rate, $Y_{e i}, s_{d}$, $S_{d}$ and $\beta_{d}$ are material parameters. The parameter values of the initial anisotropy, hardening and damage for the two materials are given in Tables 1 and 2 .

Table 1: Brass material parameters

\begin{tabular}{cccccc}
$\mathrm{n}$ & $\mathrm{k}$ & $\varepsilon_{0}$ & $\mathrm{r}_{0}$ & $\mathrm{r}_{45}$ & $\mathrm{r}_{90}$ \\
\hline 0.118 & 618.3 & 0.014 & 1.8 & 1.3 & 2
\end{tabular}

Table 2: DP steel material parameters

\begin{tabular}{ccccccccc}
$\mathrm{R}_{\text {sat }}$ & $\mathrm{C}_{\mathrm{r}}$ & $\mathrm{r}_{0}$ & $\mathrm{r}_{45}$ & $\mathrm{r}_{90}$ & $\beta_{\mathrm{d}}$ & $\mathrm{S}_{\mathrm{d}}$ & $\mathrm{s}_{\mathrm{d}}$ & $\mathrm{Y}_{\mathrm{ei}}$ \\
\hline 551.4 & 9.3 & 1 & 1.5 & 2 & 5 & 20 & 0.01 & 0
\end{tabular}

\section{LOCALIZATION CRITERIA}

Various necking and strain localization criteria are used to characterise the formability of sheets metal. The aim of this section is to present the principles and the theoretical basis of the most commonly used localization criteria. Attention is given to their respective ability to predict diffuse or localized modes as well as the orientation of a localization band.

\subsection{MAXIMUM FORCE CRITERIA}

These criteria are based on the empirical observation that necking occurs when the load reaches its maximum during a tensile test. Necking is then predicted if the load $F$ becomes stationary: $d F=0$. Extension of this diffuse necking criterion to 2D loading conditions was proposed by Swift. Using the previous condition in the two principal loading directions and combining it with the equilibrium and constitutive equations, Swift "Maximum Force Criterion" (MFC) can be obtained [1]:

$$
\frac{d \bar{\sigma}}{\bar{\sigma} d \bar{\varepsilon}}=\frac{\left(\frac{\partial \bar{\sigma}}{\partial \sigma_{1}}\right)^{2} \sigma_{1}+\left(\frac{\partial \bar{\sigma}}{\partial \sigma_{2}}\right)^{2} \sigma_{2}}{\frac{\partial \bar{\sigma}}{\partial \sigma_{1}} \sigma_{1}+\frac{\partial \bar{\sigma}}{\partial \sigma_{2}} \sigma_{2}}
$$

$\bar{\sigma}$ is the equivalent stress, $\sigma_{1}$ and $\sigma_{2}$ are the major and minor principal stresses and $\varepsilon_{1}$ the major principal strain. Extension of this principle to localized necking is given by taking into account the evolution of the strainpath change measure $\beta$, leading to the "Modified Maximum Force Criterion" (MMFC) [2]:

$$
\begin{aligned}
& \frac{\partial \sigma_{1}}{\partial \varepsilon_{1}}+\frac{\partial \sigma_{1}}{\partial \beta} \frac{\partial \beta}{\partial \varepsilon_{1}}=\sigma_{1} \\
& \beta=\frac{\dot{\varepsilon}_{2}}{\dot{\varepsilon}_{1}}
\end{aligned}
$$

Hill'52 criterion [1] is based on the same principle, but is written with an additional more restrictive condition: localization is supposed to occur in a band with stationary extension, restraining the criterion to the domain of negative minor strain. Two conditions are then obtained: one on the maximum loading and one on the orientation $\theta$ of the localization band:

$$
\frac{d \sigma_{n}}{d \varepsilon_{n}}=\sigma_{n} \quad \text { and } \quad \tan ^{2}(\theta)=-\beta
$$

\subsection{MARCINIAK-KUCZYNSKI ANALYSIS}

In this criterion [5], the strain localization is expected to occur at a defect of the structure. An initial defect (weak zone B) is introduced into a metal sheet (safe zone A). This defect is usually introduced as a band of reduced thickness, defined with the initial defect size $f_{0}$ : 


$$
f_{0}=\frac{t_{0}^{B}}{t_{0}^{A}}<1
$$

$t_{0}$ is the initial thickness in zones A and $\mathrm{B}$. This initial defect is not only a geometrical defect, but it can be seen as an equivalent defect taking into account geometrical and material heterogeneity. This parameter is a userpostulated parameter.

After loading of the sheet, mechanical properties of zones A and B are computed thanks to equations (1)-(8). Localization is predicted if the ratio of mechanical properties in zones $\mathrm{A}$ and $\mathrm{B}$, for example the cumulated plastic strain rates, exceeds a threshold $S$ :

$$
\frac{\dot{\bar{\varepsilon}}^{B}}{\dot{\bar{\varepsilon}}^{A}}>S
$$

This 2D formulation of the criterion allows the prediction of localized strain modes in the plane of the sheet and the determination of the orientation of the localization band. This criterion is easy to implement and to adapt to new material modelling; however, two non physical user-defined parameters are required.

\subsection{BIFURCATION ANALYSIS}

Another approach to predict localization phenomena is the bifurcation analysis. In this analysis, localization is seen as a bifurcation from a quasi homogeneous strain mode into a localized one.

Drucker introduced the general bifurcation criterion as a necessary condition for the loss of uniqueness of the solution to the boundary value problem for rate independent materials, excluding any elastic unloading [3]. This criterion of non-bifurcation corresponds to the positivity of the second order work:

$$
\int_{\Omega} \Delta \dot{\boldsymbol{\sigma}}: \Delta \dot{\boldsymbol{\varepsilon}} d V \geq 0
$$

For associative plasticity in small deformations, this criterion may be achieved with null or negative hardening. This criterion is a lower bound of localization, predicting the possible occurrence of the first diffuse mode. It seems to be too conservative.

Classical discontinuous bifurcation, introduced by Rice [4], is a special case of the general bifurcation, associated with kinematically admissible discontinuous modes. After introducing a discontinuity into the velocity gradient, the localization criterion takes the form:

$$
\begin{aligned}
& \mathbf{Q}=\mathbf{n} \cdot \mathbf{L} \cdot \mathbf{n} \\
& \operatorname{det}(\mathbf{Q})=0
\end{aligned}
$$

In the case of associative plasticity, this criterion requires negative hardening to be activated. This effect is introduced by the use of damage. This criterion always occurs after general bifurcation and predicts 3D localization modes as well as the band orientation.
However, it requires damage and cannot be used with rate sensitive materials.

\subsection{STABILITY ANALYSIS BY LINEAR PERTURBATION METHOD}

Localization can be seen as the development of unstable deformation modes with high growing rates. The linear perturbation method [6] can be used to check the stability of the equilibrium equations. The equilibrium problem can take the form of a set of equations written under the form:

$$
\mathbf{A}(\mathbf{U})=\mathbf{0}
$$

with $\mathbf{U}$ a vector containing the strain rate tensor, the equivalent plastic strain rate, the stress tensor and the equivalent stress. The solution $\mathbf{U}_{0}$ of the system is perturbed by small perturbations of assumed form:

$$
\mathbf{U}=\mathbf{U}_{0}+\delta \mathbf{U}=\mathbf{U}_{0}+\delta \mathbf{U}_{0} e^{\eta t}
$$

where $\delta \mathbf{U}_{0}$ is the amplitude and $\eta$ the growing rate of the perturbation. Perturbed system takes the form:

$$
\begin{aligned}
& \mathbf{A}\left(\mathbf{U}_{0}, \eta\right) . \delta \mathbf{U}_{0}=\mathbf{0} \\
& \operatorname{Det}\left(\mathbf{A}\left(\mathbf{U}_{0}, \eta\right)\right)=0
\end{aligned}
$$

Solution of this eigenvalue problem leads to the resolution of a third order polynomial in $\eta$.

Eigenvalues with negative real parts correspond to stable modes. Eigenvalues with positive real parts and low growing rates correspond to diffuse necking while those with growing rate superior to the equivalent plastic strain rate are assumed to be localized.

This method allows the determination of diffuse and localized modes as well as the orientation of localization band and is also suitable for rate sensitive materials. In this paper, a 2D formulation of this criterion is implanted, allowing the determination of modes in the plane of the metal sheet.

\section{FORMABILITY OF METAL SHEETS}

The FLDs predicted with Swift, Hill, Hora, M-K and Molinari localization criteria for a brass without damage and with M-K and Rice for a DP steel with damage are compared respectively in Figure 1 and in Figure 2.

In Figure 1, prediction of diffuse necking modes by the Swift and Molinari criteria gives close results. In the case of localized modes, the results are more dispersed. The M-K model seems to lower the formability in plane tension and to overestimate it in equibiaxial tension. This general trend can be adjusted by the user imposed parameters. Within the stability analysis, modes found in the plane of the sheet overestimate the formability. They can be interpreted as an upper localization bound. A 3D formulation of this criterion would allow the search of modes out of the plane of the sheet and would be more suitable for this application. 


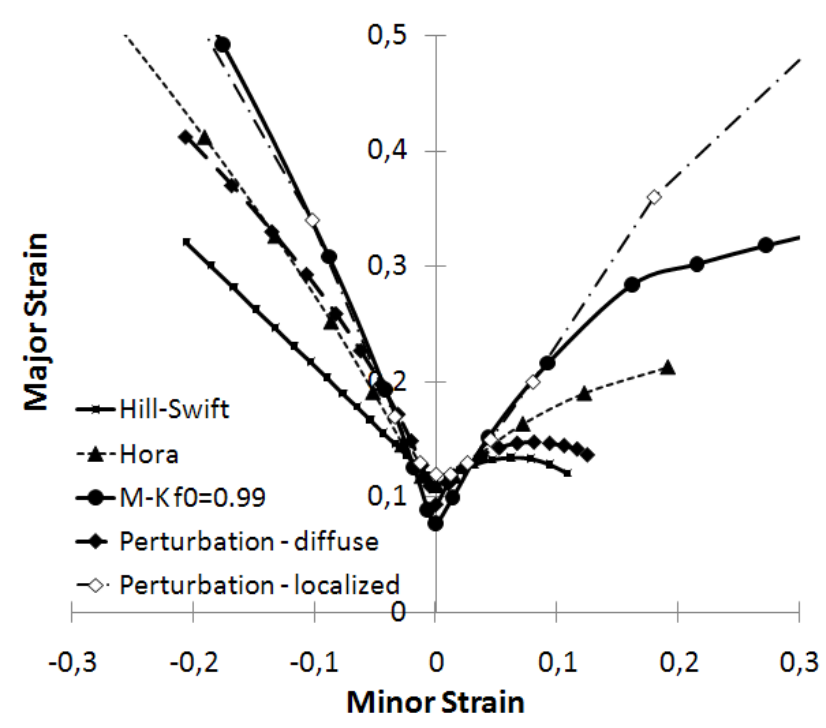

Figure 1: FLDs of a brass

Figure 2 shows the FLD predicted by the M-K and the Rice models for a DP steel. Within associative plasticity, Rice criterion requires softening, which is introduced here by damage. Trends mentioned above with M-K criterion are still recovered. Parametric analysis is carried out on the size of the initial defect $f_{0}$. Prediction of M-K criterion with small initial defect $\left(f_{0} \approx 1\right)$ tends to reproduce the FLD obtained with Rice criterion. With large initial defect $\left(f_{0} \approx 0.95\right)$, formability is lowered and the FLD could be closer to a diffuse mode.

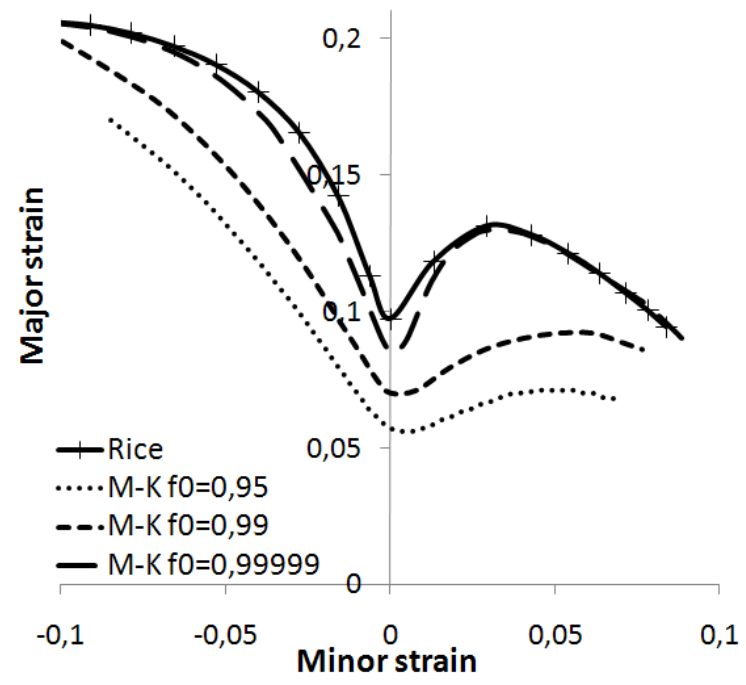

Figure 2: FLDs of a DP steel

\section{CONCLUSIONS}

In this paper, theoretical formulations of commonly used localization criteria are reviewed. Applicability domains of the criteria are reviewed, along with their advantages and drawbacks.
Coupling with a general class of material modelling allows the simulation of forming limit diagrams. Comparison of numerical FLD allows the verification of theoretical discussion and reveals some trends. Diffuse modes can be predicted with Swift Maximum Force and Molinari stability analysis criteria; predictions obtained with these criteria are in good accordance. Localized modes are predicted by Hill, Hora, Rice, M-K and Molinari criteria. Results are more dispersed. Stability analysis with localized mode, M-K with small initial defect and Rice criteria seem indicate an upper bound of formability, while M-K with large initial defect and Hora criteria seem give intermediate modes.

Further developments are concerned with the coupling of the localization criteria with physically based constitutive laws in order to improve the FLD simulation and to compare experimental and numerical results.

\section{ACKNOWLEDGEMENT}

The first author is financially supported by the French CNRS and the Région Lorraine and is grateful to Dr. A. Lejeune, LPMM, University of Metz, for fruitful scientific discussions.

\section{REFERENCES}

[1] Hill R.: On discontinuous plastic states, with special reference to localized necking in thin sheets. J. Mech. Phys. Solids, 1:19-30, 1952.

[2] Hora P., Tong L., Reissner J.: A prediction method of ductile sheet metal failure in FE simulation. 3rd International Conference: Numisheet '96, 1996. The Ohio State University.

[3] Drucker D.C.: Some implications of work hardening and ideal plasticity. QAM, 7:411-418, 1950.

[4] Rice J.R.: The localization of plastic deformation. In: $14^{\text {th }}$ IUTAM Congress, 1976. Amsterdam.

[5] Marciniak Z., Kuczynski K.: Limit strains in the processes of stretch-forming sheet metal. Int. J. Mech. Sci., 9:609-620, 1967.

[6] Dudzinski D., Molinari A.: Perturbation analysis of thermoplastic instabilities in biaxial loading. Int. J. Solids Struct., 27:601-628, 1991.

[7] Lejeune A., Boudeau N., Gelin J.C.: Influence of material and process parameters on bursting during hydroforming process. J. Mater. Proc. Technol., 143:11-17, 2003.

[8] Haddag B.: Contribution à la modélisation de la mise en forme de tôles métalliques : application au retour élastique et à la localisation. $\mathrm{PhD}$ thesis, Arts et Métiers ParisTech, Metz, France, 2007. 